\title{
The Changing Context of Education: The Need for Interprofessional Education Teaching
}

\author{
Mary J Huber ${ }^{1 \star}$, Barbara Fowler ${ }^{2}$ \\ ${ }^{1}$ Professor at Wright State University \\ ${ }^{2}$ Professor in Nursing at Wright State University
}

\author{
${ }^{*}$ Corresponding author \\ Mary J Huber, Professor at Wright State University. \\ Submitted: 03 Jul 2020; Accepted: 15 Jul 2020; Published: 20 Oct 2020
}

\begin{abstract}
As the pedagogy of higher education in health care fields and human services has evolved over the past decade the need for interprofessional education (IPE) between "two or more professions learning about, from and with each other to enable effective collaboration and improve health outcomes" for society has come to the forefront [1]. Interprofessional higher education aims to teach students boundary-crossing skills that integrate knowledge from two or more disciplines to produce a cognitive advancement in ways that would be impossible if students are taught by soloed disciplines [1]. Students who become healthcare providers often are educated side-by-side but with little interaction between each other [2]. Increasing the link between student's education and clinical practice can allow for more interaction between multidisciplinary health fields, with the goal of fostering interprofessional collaboration in the long-term. This study involved students from two disciplines, nursing and counseling, attending a community event together. Afterword, a focus group was held to examine how the students learned from each other and how they felt about the event which was a twohour workshop for persons addicted to opioids and their friends and family. Overwhelmingly, the qualitative data gathered from the focus group demonstrated that the students learned from each other and enjoyed the experience.
\end{abstract}

The Changing Context of Education: The Need for Interprofessional Education Teaching

As the pedagogy of higher education in health care fields and human services has evolved over the past decade the need for interprofessional education (IPE) between "two or more professions learning about, from and with each other to enable effective collaboration and improve health outcomes" for society has come to the forefront [1]. Interprofessional higher education aims to teach students boundary-crossing skills that integrate knowledge from two or more disciplines to produce a cognitive advancement in ways that would be impossible if students are taught by soloed disciplines [1]. Students who become healthcare providers often are educated side-by-side but with little interaction between each other [2]. Increasing the link between student's education and clinical practice can allow for more interaction between multidisciplinary health fields, with the goal of fostering interprofessional collaboration in the long-term.

Numerous authors have recognized the need for higher education to shift from silos to IPE [3, 4]. IPE takes into account the need for approaches from multiple disciplines and health professions in order to address complex clinical problems $[5,6]$. In this transition to learning, students benefit from a shift from learning in silos in traditional pedagogical education settings to innovative approaches that support IPE in communities [6,7].

In 2011, the Robert Wood Johnson Foundation (RWJF) issued a Workforce Issue Brief reporting that IPE prepare health professional students to take on leadership roles in assisting individuals in accessing healthcare services and improving the quality of care in changing, complex health systems. The needs of consumers have also changed emphasizing the importance of IPE. More and more communities are providing community-based interventions such as health screenings, health promotion, and health literacy education requiring professionals to have a broad education across disciplines. In 2003, the Institute of Medicine (IOM) released a hallmark report on Health Professions Education: A Bridge to Quality. The report emphasized that health education may incorporate different settings or situations including in the classroom, online, or within local communities for the benefit of the community. IPE in communities provide unique opportunities for students to learn about the strengths, needs, resources as well as problems and strategies that may be relevant to improving the health of the community [7]. IPE in communities represents a more equal relationship between experts and learners, as opposed to the more traditional approach of learning between teachers and 
students in traditional education settings [8]. Spelt, Harm, Biemans, Pieternel, and Luning [9]. assert that IPE instruction that promotes collaborative thinking creates and fosters a higher level thinking.

Since its inception, several organizations, including the WHO and the American Association of Colleges of Nursing (AACN) emphasized that IPE is vital to the learning needs of students, particularly in light of the rapid changing healthcare system and patient population impacted by health disparities and language barriers. The WHO Framework for Action emphasized that IPE can assist students in learning about, from, and with each other in achieving better learning experiences in various settings. Comparatively, the AACN Essentials of Baccalaureate Education for Professional Nursing Practice (2008) addressed the benefits of IPE and collaboration between professionals in Essential VI. The IOM report (2003) and Essential VI highlighted that IPE and collaboration "are vital in addressing patient problems and social determinants of health (erosion of neighborhoods, limited or meager financial resources, and lack of access to available and affordable healthcare) to improve the overall quality of care for populations served".

A benefit of providing healthcare in communities is that consumers benefit from interactions with students as well as potentially increasing the linkages with available community resources (housing, food and clothing, healthcare, support networks) and providers [10]. In addition, students learn from community members the extent to which gaps in services affect their health and well-being as well as gain insight into the dynamics of the decision-making structures (local legislators, leaders in churches or places of worship, schools, and neighborhood watch programs) and healthcare system within communities. Finally, implementing IPE in a community calls for increased attention to the importance of establishing partnership with people who live or work in the community. These partnerships between University faculty, students, and community agencies and/or provider's builds bridges within the community, while at the same time closing gaps in healthcare education and community needs.

While there is clear evidence supporting IPE across health disciplines, there are also several barriers that interfere with IPE. In separate reviews, King and colleagues and Lawlis, Anson, and Greenfield reported individual-level and institutional barriers to IPE between professionals, such as scheduling demands in an already demanding curriculum, creation and implementation of learning experiences, and specific scenarios that stimulate interest among different groups of students. Lawlis et al. noted that variable length of programs, different curriculum requirements, disparity in the number of students within each program, and institutional funding were hindrance to IPE. Comparatively, the sustainability of IPE is affected by factors, such as knowledge, interest, buy-in, and enthusiasm of faculty support [11-14].

As educators develop strategies for IPE, administrators must understand that barriers, such as shifts in organizational priorities and lack of understanding of time commitment may unintentionally affect the success of IPE. Furthermore, useful resources such as technology, space associated with collaboration, and time commitment of educators must be underscored in promoting opportunities for learning experiences of students across professions [5-15]. Other important factors influencing the success of IPE is shared interprofessional vision and "champions" across professions that reflect attitudes of success and team players [16, 17]. Below is a description of the implementation of IPE in a community struggling with the opioid epidemic.

\section{Our Beginning}

Wright State University is a moderate-size, teaching intensive university in the Midwest. The university has numerous educational resources supporting the teaching, scholarship and service commitment of faculty. In addition, the university's mission statement strongly supports collaboration and IPE, practice partnerships and multidisciplinary activities between faculty and communities served. We therefore, seized the opportunity to provide a unique joint collaborative learning experience for online graduate students in two separate programs. Students were enrolled in the Population Health course in the College of Nursing and Health $(\mathrm{CONH})$ and the Treatment of Addictions course in the College of Education and Human Services (CEHS). Both faculty are currently involved in varying practice partnerships and service activities supporting the health and well-being of underserved and medically-uninsured populations. At the hub of the population served are persons and their families as well as support networks affected by Opioid Use Disorder (OUD). In addition, we are identified as "champions" of student learning and interested in ensuring that students are exposed to different learning experiences in communities served by both professions. We also believed that creating this learning experience would produce further opportunities for IPE and collaboration with a broader range of professionals (social work, public health, education and medicine) to name a few and benefit the population served. The first author of this paper has played an important role in the development, ongoing implementation and evaluation of a community event, referred to as Conversation for Change (C4C). Hallmark of the learning experience was attendance at the community event.

\section{Overview of Conversations for Change}

$\mathrm{C} 4 \mathrm{C}$ is a community-based opioid harm reduction program that uses Evidence-Based Practices (EBP) to assist individuals with Opioid Use Disorder (OUD), their families and friends to find local resources and support for their treatment and recovery. The term 'treatment' is defined broadly here to include inpatient, outpatient, Medication-Assisted Treatment (MAT), and Certified Peer Support (CPS). It is an interactive event where local police, multi-sector partnerships, and people in recovery gather to provide a safe place for community members struggling with addiction to come and learn about local resources that are available to them. This program was modeled after national programs including the Boston Gun Project's Operation Ceasefire the Law Enforcement Assisted Diversion (LEAD) program (n.d.), and the Angel program [18]. 
The event takes place in a 'hot-spot' area in Montgomery County in Dayton, Ohio; an area where opioid mortality rates are both high and rapidly increasing, according to an analysis by the Centers for Disease Control and Prevention [CDC] (2011) [19, 20]. Dayton is a moderate-size urban city that has experienced the highest rates of deaths and overdoses from the opioid epidemic [21-25].

The two-hour event includes a hot meal, information on local resources, Narcan training, treatment options, recovery stories, and individualized conversations between the participant and a professional trained in Motivational Interviewing (MI) to discuss personal needs and recovery options. According to the American Addiction Centers (2018) and others, MI techniques have shown to be very effective in working with individuals struggling with addiction [26-28]. Central to the event is the MI conversation and training on the administration of Narcan. Despite C4C being in its fifth year, it is still considered a pilot program due to its everchanging and evolutionary nature. Throughout the first few years of implementing $\mathrm{C} 4 \mathrm{C}$, feedback was received from participants and utilized to continuously improve and address the participants' needs. Despite limited resources to gather data to examine the effectiveness of $\mathrm{C} 4 \mathrm{C}$ in helping individuals recover from OUD, a short evaluation survey was created and administered after each two-hour event.

The purpose of the current study is to explore the experiences and views of nursing and counseling students who participated in an IPE community event. The questions that guided this effort were:

1. How would the attendance and observation of a $\mathrm{C} 4 \mathrm{C}$ event affect the perceptions of opioid addiction among graduate students enrolled in either nursing or in a chemical dependency counseling program?

2. How would the attendance and interaction between these two groups of students during and after the event affect their overall learning experience?

3. Would the comfort level in working with individuals with OUD change after attending $\mathrm{C} 4 \mathrm{C}$ ?

\section{Method}

\section{Study Design}

Two semesters of students were invited to participate in the IPE learning experience. Both faculty members announced or advertised the $\mathrm{C} 4 \mathrm{C}$ program on the course welcoming page or their syllabus to their perspective students. The announcement included a statement that the number of students was limited to 6-10 students in each discipline so students were selected by faculty in the respective course on a first come basis. Due to the increased number of interested students from both disciplines, some students did not attend but were expected to complete a different assignment not linked to the $\mathrm{C} 4 \mathrm{C}$ learning experience.

The students and faculty members met at the location of the $\mathrm{C} 4 \mathrm{C}$ event before it started. Introductions were done and the agenda for the next two hours was explained. Students were informed that our purpose at $\mathrm{C} 4 \mathrm{C}$ is not to make value judgements, but to learn about OUD from the community's perspective. The group then observed the event and met again after the event to discuss immediate reactions. At the end of this discussion days for a focus group were presented and decided. The focus group, scheduled for approximately 1 hour, occurred about two weeks following the event. Prior to the start of the focus group, the students signed a consent form to participate in the focus group. The following questions were sent out to the students prior to the focus group meeting. These questions mirror the research questions stated above related to student's perception of individuals with OUD, student's interactions with each other, and student's comfort level while attending the event and their feelings about working with people with OUD in the future. Students were instructed to use the questions as a guide to preparing for the focus group: What were your perceptions of individuals with OUD before and after attending the C4C event? What did you learn from students who are in a different area of study? Describe your comfort level in working with individuals with OUD before and after attending C4C.

\section{Results}

Two forms of data addressed the research questions: data from the focus group and perception papers that were written by each student after attending the event and focus group. The total number of nursing students over the two semesters was 16 and the total number of counseling was 8 . Both faculty members reviewed the data separately, then together to compare commonalities and different views between the students. Students from both disciplines valued the opportunity to share their experiences in working with patients with OUD. In addition, students provided insightful comments about IPE ranging from self-reflection to statements about learning from each other as well as learning from the community environment. The events occurred in the basement of a local church, similar to a community hub for local residents surrounded by blight (broken windows, boarded up buildings) and meager resources. Several students informally addressed the issue of visiting a different setting from their comfort zone of the local hospital setting or university setting. A few nursing students shared with the faculty prior to $\mathrm{C} 4 \mathrm{C}$ that they work at a hospital nearby the $\mathrm{C} 4 \mathrm{C}$ events but never spend time in the community. Another commonality was that some nursing students visited the community prior to $\mathrm{C} 4 \mathrm{C}$ to better understand the consequences of OUD, such as poverty and neighborhood decay.

Different themes were developed from the data. A common theme was that students felt that working with students from another discipline was extremely helpful.

One student wrote: In the future, I think that pairing up with a student from each discipline, even for a short time, could be an interesting way to promote dialogue, even if it was just through email or a discussion board conversation throughout the semester.

\section{Similarly}

Coming together through projects like this opens channels of communication and affords students an opportunity to understand 
things from a perspective they may not otherwise consider. Still another student stated: Nurses see the same patients continually coming back from multiple overdoses. I believe working with those students (counseling) was a great opportunity. Working with them helps complete the circle of the addiction field. While both programs may be too broad with their own set of learning experiences, there is room for essential elements to be incorporated into both curriculums and programs of study. Another major theme was the learning that took place in the community setting.

One student wrote: The opportunity of engaging in the community was invaluable because other than public health education experiences, there are shortcomings of traditional nursing education programs that train nurses to provide care or function at the bedside rather than function in complex social situations.

Another student stated: The $\mathrm{C} 4 \mathrm{C}$ program provided a great crossover experience for nursing students and those in rehabilitation counseling to broaden our experiences with clients experiencing opioid addiction, while at the same time learning about or understanding the shortcomings of the ecosystem that contributes to the promotion of drug addiction.

As described by another student: We need a facilitating onestop-shop of sorts that would have the availability of services, requirements for services, access to transportation to and from services, and a relationship with providers for appointment setting. This entity would have the basis for community collaboration and would maintain up-to-date agency information, that referrals could be effectively achieved.

Finally, another student stated: It was extremely beneficial to have the beginning of a rolodex to take back to the workplace to disseminate information learned with other nurses or co-workers who may be unaware of the numerous programs available for patients and their families served in the community. To me, learning experiences like this are likely to grow as healthcare continues to shift away from acute care to a community model that is incredibly focused on prevention.

\section{Discussion}

The WHO Framework for Action (2010) highlighted the benefits of engaging students from different disciplines and learning about, from, and with each other in achieving better patient care and improving public health [1]. More important, IPE helps students gain insight into the views of others that are essential for building collaborative relationships and working with the community. It was very apparent that nursing students are taught from the medical model and view substance abuse treatment as procedural rather than individualistic. This was underscored by one nursing student who used the word 'hardening' to describe a process that can happen to nurses who only see patients in the emergency room or in critical care. Hardening, in this case, is defined as an almost unsympathetic outlook toward individuals with OUD. Although many of the nursing students shared the feelings of being 'hardened' to these situations, they seemed interested in incorporating some type of counseling, including active or focused listening into their training and openness to changing their perspective. Interestingly, a nursing student even noticed the manner in which the counseling students spoke of their clients who have OUD in a calmer less irritated voice than did the nursing students adding that interacting with students in the counseling program was very insightful. During the focus group, there appeared to be a shift in perspectives and each group was able to view addiction in a different lens.

When students came together for the first time, they initially positioned themselves based on their areas of expertise (educational preparation, work experiences or work setting), later they began to share common concerns and in some instances, deeply held negative views about working with or caring for persons/patients with OUD. As the interaction continued, students shifted to expressing concerns about how opioid use is paralyzing to individuals and their families or support network. For example, some students expressed disappointment in family members or patient's slow recovery in OUD. Their willingness to share demonstrated the benefit of participating in C4C learning. Students became empowered from the IPE learning experience by gaining knowledge and new insights in terms what each discipline contributed in caring for persons addicted to opioids. Therefore, collaboration with students from different disciplines was enriching and beneficial to future professional development. The data from this study supports this assertion. Other benefits centered on working together to learn from the community about the growing problem of Opioid Addiction in Montgomery County, Ohio.

Another theme that appeared in the data was the awareness of so many community partners who participated in the $\mathrm{C} 4 \mathrm{C}$ event and the feeling that the providers really care about the wellbeing of the participants. This seemed to be a surprise to many of the students, in particular the nursing students. Many students in both groups were made aware of the plethora of addiction, rehabilitation and support resources available to people in the Dayton area. One student summed it up by saying "I had been previously unaware of so many services and support groups in the community, ranging from those in recovery to law enforcement working to treat addiction as a disease rather than a crime." The anecdotal information gathered from students during the focus groups was overwhelmingly positive and many of the students were grateful for having the opportunity to attend the event and learn from each other. After the event, most students stated they would inform other professionals as well as family members of the vast opportunities available and community support for persons with OUD in Dayton, Ohio.

\section{Implications for Research, Practice and Teaching}

Spelt et al., and Hammick et al., showed that, to date, scientific research into teaching and learning in interprofessional higher education has remained limited and explorative. The research advanced the understanding of the necessary subskills of interprofessional thinking and typical conditions for enabling the 
development of interprofessional thinking. This understanding provides a platform from which the theory and practice of interprofessional higher education can move forward [3-9].

The IPE model is a novel method to training and educating the future workforce allowing for differing views to be expressed and different disciplines of students to learn from each other. There is a need to invest in a comprehensive teaching approach to reduce substance abuse. CDT is a technique that has some evidence to support its effectiveness in integrating learning experiences but needs more research [29]. One might be excused for seeing the IPE teaching method as having a minimal influence on student learning experience and professional development. However, given the positive feedback that was received in this study, it is suggested that IPE can have lifelong impact on a person's professional career. Therefore, teaching in silos should be abandoned and innovative approaches such as IPE are recommended.

The results from this study can inform further studies, for example the creation and use of surveys to gain additional knowledge about the medical model approach to OUD in communities. Survey data could help determine the prevalence of any given attitude or experience of the participants and could facilitate the integration of qualitative and quantitative methods in order to more comprehensively identify and address potential changes to these attitudes. The potential for further collaboration and IPE between professionals and communities are vast, especially in gaining new insights in meeting the healthcare needs of individuals and their families and support systems experiencing opioid addiction. Partnerships could be established with faculty in other professions supporting the learning needs of medical students and those in other professions, such as the School of Professional Psychology. Furthermore, undergraduate students in nursing and other disciplines could be exposed to IPE and the plethora of learning opportunities in meeting the healthcare needs of communities affected by OUD. In conclusion, rigorous evaluation of IPE efforts must be supported and include qualitative and quantitative elements.

\section{Study Limitations}

The qualitative nature of this study and the small number of study participants limits the generalizability of the findings; however, the benefits of the IPE between two different professionals support the student's learning in IPE [30-54].

\section{References}

1. World Health Organization (2010) Framework for Action on Interprofessional Education and Collaborative Practice. Hopkins, D. (Ed.). Geneva, Switzerland 2010: 6-62.

2. Spector N (2010) Collaboration: A nursing perspective. In B Freshman, L Rubino, Y Reid-Chassiakos (Eds.) Collaboration across the disciplines in health care (pp. 107-132). Jones and Bartlett Publishers, LLC.

3. Hammick M, Freeth D, Koppel I, Reeves S, Barr H (2007) A best evidence systematic review of interprofessional education: BEME Guide, No.9. Medical Teacher 29: 735-751.
4. Speakman E, Collins L, Arenson C, Sicks S (2015) From the Editors, Collaborative Healthcare: Interprofessional Practice, Education and Evaluation 6: 1.

5. Begg MD, Crumley G, Fair AM, Martina CA, Mc Cormack WT, et al. (2014) Approaches to preparing young scholars for careers in interdisciplinary team science. Journal of Investigative Medicine: The Official Publication of the American Federation for Clinical Research 62: 14-25.

6. Croyle RT(2008) The National Cancer Institute's transdisciplinary centers initiatives and the need for building a science of team science. American Journal of Preventive Medicine 35.

7. Kothari A, Boyko JA, Conklin J, Stolee P, Sibbald SL (2015) Communities of practice for supporting health system change: a missed opportunity. Health Research Policy System 13.

8. Li LC, Grimshaw JM, Nielsen C, Judd M, Coyte PC, et al. (2009) Evolution of Wenger's concept of community of practice. Implement Science 4.

9. Spelt EJH, Harm JA, Biemans HT, Pieternel A, Luning MM (2009) Teaching and learning in interdisciplinary higher education: A systematic review. Educational Psychology Review 21: 365-378.

10. Stanhope M, Lancaster J (2019) Public health nursing: Population-centered healthcare in the community $\left(10^{\text {th }}\right.$ ed.). Elsevier Health Sciences.

11. Blue AV, Mitchum M, Smith T, Raymond J, Greenberg R (2010) Changing the future of health professions: Embedding interprofessional education within an academic health center. Academic Medicine 85: 1290-1295.

12. Lawlis TR, Anson J, Greenfield D (2014) Barriers and enablers that influence sustainable interprofessional education: A literature review. Journal of Interprofessional Care 28: 305-310.

13. Mitchell PH, Belza B, Schaad DC, Robins LS, Gianola FJ, et al. (2006) Working across the boundaries of health professions disciplines in education, research, and service: The University of Washington experience. Academic Medicine 81: 891-896.

14. McNair R, Brown R, Stone N, Sims J (2001) Rural interprofessional education: Promoting teamwork in primary health care education and practice. Australian Journal of Rural Health 9: 19-26.

15. Clark PG (2011) Examining the interface between interprofessional practice and education: Lessons learned from Norway for promoting teamwork. Journal of Interprofessional Care 25: 26-32.

16. Barker K, Bosco C, Oandasan IF (2005) Factors in implementing interprofessional education and collaborative practice initiatives: Findings from key informant interviews. Journal of Interprofessional Care 19: 166-176.

17. Freeth D (2001) Sustaining interprofessional collaboration. Journal of Interprofessional Care 15: 37-46. 
18. Schiff DM, Drainoni ML, Bair-Merritt M, Rosenbloom D (2016) A police-led addiction treatment referral program in Massachusetts. The New England Journal of Medicine 375: 2502-2503.

19. Centers for Disease Control and Prevention(2017) Understanding the epidemic. https://www.cdc.gov/drugoverdose/epidemic/ index.html

20. Weisburd DL, Groff ER, Morris N (2011) Hot spots of juvenile crime: Findings from Seattle, Juvenile Justice Bulletin, U.S. Department of Justice, OJJDP.

21. Carlson RG, Li L, Daniulaityte R (2017) Montgomery County Poisoning Death Review: 2010-2016. (Dayton, OH: Center for Interventions, Treatment \& Addictions Research), https:// www.phdmc.org/epidemiology/poisoning-death-review/677montgomery-county-poison-death-review-report-2017/file.

22. Democracy Collaborative (2016) Dayton, Ohio. https:// communitywealth.org/content/dayton-ohio .

23. Quinones S (2016) Dreamland: The true tale of America's opiate epidemic. New York, NY: Bloomsbury Press.

24. Sewell D (2018) New surge of meth, cocaine mixed with powerful opioid pushes Ohio's drug overdose death toll higher. https://www.ohio.com/akron/news/breaking-news-news/ new-surge-of-meth-cocaine-mixed-with-powerful-opioidpushes-ohios-drug-overdose-eath-toll-higher.

25. Staley SR (2008) Dayton, Ohio: The rise, fall, and stagnation of a former industrial juggernaut, New Geography Blog, http://www.newgeography.com/content/00153-dayton-ohiothe-rise-fall-and-stagnation-a-former-industrial-juggernaut.

26. American Addiction Centers (2018) Motivational interviewing in addiction treatment. https://americanaddictioncenters.org/ therapy-treatment/motivational-interviewing/.

27. Ilgen MA, Glass JE (2013) Brief motivational interventions to change problematic substance use. In MG Vaughn and BE Perron, (Eds). Social work practice in the addictions. New York: Springer.

28. Miller WR \& Rollnick S (2002) Motivational interviewing: Preparing people for change (2nd ed.). New York: Guilford Press 25: 46.

29. Weaver T (2008) Enhancing multiple disciplinary teamwork. Nursing Outlook 56: 108-114.

30. American Association of Colleges of Nursing: (2008) The essentials of baccalaureate education for professional nursing practice (2008). Washington, DC: One Dupont Circle, Suite 530. http://www.aacn.nche.edu/Education/pdf/BaccEssentials08.pdf.

31. Frankel TC (2015) Pellets, planes and the new frontier: How Mexican heroin cartels are targeting small-town America" https://www.washingtonpost.com/sf/national/2015/09/24/ pellets-planes-and-the-new-frontier/? utm term $=.845 \mathrm{a} 51 \mathrm{f} 7 \mathrm{~b} 6 \mathrm{a} 1$.
32. Goodnough A (2018) This city's overdose deaths have plunged. Can others learn from it? https://www.nytimes. com/2018/11/25/health/opioid-overdose-deaths-dayton.html

33. Greiner AC, Knebel E (2003) Health professions education: A bridge to quality. Institute of Medicine (US) Committee on the Health Professions Education Summit.

34. Washington, DC: National Academies Press.

35. Hall P (2005) Interprofessional teamwork: Professional cultures as barriers. Journal ofInterprofessional Care 19: 188196.

36. Harris JL, Roussel L, Dearman C, Thomas PL (2020) Project planning and management: A guide for nurses and interprofessional teams $\left(3^{\text {rd }}\right.$ ed.). Burlington, MA: Jones \& Bartlett Learning.

37. King S, Hall M, McFarlane LA, Paslawski T, Sommerfeldt S, et al. (2017) Launching first-year health sciences students into collaborative practice: Highlighting institutional enablers and barriers to success. Journal of Interprofessional Care 31: 386393.

38. Kreitner R, Kinicki A (2008) Organizational behavior (8th ed.). Boston: McGraw Hill/Irwin.

39. Lang A (2017) New data shows drug overdoses increased in 40 states and Washington, D.C. http://healthyamericans.org/ newsroom/releases/?releaseid $=376$

40. LEAD: Law Enforcement Assisted Diversion. (n.d.). About LEAD. Retrieved from http://leadkingcounty.org/about/

41. Macy B (2019) Dopesick: Dealers, Doctors, and the Drug Company that Addicted America. New York, NY: Hatchette Book Group, Inc 32: 466-467.

42. Marcelo P (2016) Researchers: Gloucester's Angel program helped nearly 400 drug addicts. http://boston.cbslocal. com/2016/12/21/gloucester-police-angel-program-helped400-drug-addicts/

43. Miller M (2015) The relevance of twelve-step recovery in $21^{\text {st }}$ century addiction medicine. American Society of Addiction Medicine. https://www.asam.org/resources/publications/ magazine/read/article/2015/02/13/the-relevance-of-twelvestep-recovery-in-21st-century-addiction-medicine

44. National Drug Control Strategy (2019) A Report by the Office of National Drug Control Policy.

45. National Institute on Drug Abuse (2018) Opioid overdose crisis. https://www.drugabuse.gov/drugs-abuse/opioids/ opioid-overdose-crisis

46. Neergaard MA, Olesen F, Andersen RS, Sondergaard J (2009) Qualitative description - The poor cousin of health research? BMC Medical Research Methodology 9: 52.

47. Nelson L, Schwaner R (2009) Transdermal fentanyl: Pharmacology and toxicology. Journal of Medicine Toxicology 5: 230-241. 
48. Ohio Department of Health (2018) Fentanyl and related drugs overdose deaths. http://publicapps.odh.ohio.gov/EDW/ DataBrowser/Browse/Mortality

49. Public Health Dayton \& Montgomery County (2018) Accidental overdose death totals. http://www.phdmc.org/ coat/158-accidental-overdose-death-totals.

50. Rembert M, Betz M, Feng B, Partridge M (2017) Taking measure of Ohio's Opioid crisis. Swank Program in RuralUrban Policy. https://cpb-us-w2.wpmucdn.com/u.osu.edu/ dist/2/14548/files/2017/10/SWANK-Taking-Measure-ofOhios-Opioid-Crisis-1vtx548.pdf

51. Robert Wood Johnson Foundation (2011) What can be done to encourage more interprofessional collaboration in healthcare? Health Policy Snapshot. Workforce.
52. Schwartz RP, Gryczynski J, O'Grady KE, Sharfstein JM, Warren G, et al. (2013) Opioid agonist treatments and heroin overdose deaths in Baltimore, Maryland, 1995-2009. American Journal of Public Health 103: 917-922.

53. US. Department of Justice (2001) Reducing gun violence: The Boston gun project's operation ceasefire. Retrieved from National Criminal Justice Reference Service. https://www. ncjrs.gov/pdffiles1/nij/188741.pdf

54. Welch E (2019) A community of recovery: Dayton, Ohio's compassionate, collective approach to the opioid crisis. https://www.americanprogress.org/issues/criminal-justice/ reports/2019/01/10/464889/a-community-of-recovery/
Copyright: @2020 MaryJ Huber. This is an open-access article distributed under the terms of the Creative Commons Attribution License, which permits unrestricted use, distribution, and reproduction in any medium, provided the original author and source are credited. 